\title{
Relación entre la procrastinación académica y ansiedad-rasgo en estudiantes universitarios pertenecientes al primer año de estudios de una universidad privada de Lima Metropolitana.
}

Relationship between academic procrastination and anxiety-trait in university students belonging to the first year of studies of a private university from Metropolitan Lima.

\author{
Susana Elizabeth Mamani Guerra ${ }^{1}$, Ana Aguilar Angeletti ${ }^{1}$
}

\section{RESUMEN}

La finalidad de la presente investigación fue determinar si existe relación entre la procrastinación académica y la ansiedad rasgo en 200 estudiantes universitarios pertenecientes al primer año de estudios de una universidad privada de Lima Metropolitana. De igual manera, se determinó la correlación entre las dimensiones de la procrastinación académica; autorregulación académica y postergación de actividades con ansiedad rasgo. Además, se realizó un análisis comparativo de la procrastinación académica en relación a la edad, sexo, composición familiar, lugar de procedencia, facultad y número de cursos desaprobados. Se utilizó la Escala de Procrastinación Académica y el Inventario de Ansiedad Rasgo-Estado. Se encontró correlaciones de tipo muy débil entre la procrastinación académica y ansiedad rasgo $(\mathrm{r}=.068)$, autorregulación académica y ansiedad rasgo $(\mathrm{r}=.152)$ y postergación de actividades y ansiedad rasgo $(\mathrm{r}=.158)$. No se encontró diferencias significativas según las variables de comparación en la dimensión de postergación de actividades, en la dimensión de autorregulación académica sólo se halló diferencias significativas según sexo.

PALABRAS CLAVE: Procrastinación académica, ansiedad rasgo, estudiantes universitarios.

\section{SUMMARY}

The purpose of the present investigation was to determine the correlation between academic procrastination and trait anxiety in 200 university students belonging to the first year of studies of a private university of Metropolitan Lima. Similarly, it was to determine the correlation between the dimensions of academic procrastination: academic self-regulation and postponement of activities with trait anxiety. In addition, it was perfomed an analysis of levels of procrastination in relation to age, sex, family composition, origin, faculty and courses disapproved. The Academic Procrastination Scale and the State-Trait Anxiety Inventory were used. We found very weak correlations between academic procrastination and trait anxiety $(\mathrm{r}=.068)$, academic self-regulation and trait anxiety $(\mathrm{r}=.152)$ and postponement of activities and trait anxiety $(\mathrm{r}=.158)$. There were no significant differences between the variables of comparison and the dimension of postponement of activities, in the autoregulation dimension, differences are found only regarding the gender variable.

KEYWORDS: Academic procrastination, trait anxiety, university students.

\footnotetext{
Facultad de Psicología, Universidad Peruana Cayetano Heredia. Lima, Perú.
} 


\section{INTRODUCCIÓN}

La vida universitaria se caracteriza por ser un periodo sumamente competitivo debido a las constantes exigencias académicas. Esta experiencia significa, para los estudiantes, la primera evaluación entre sus proyectos y la expectativa de la realidad; así pues, los hábitos y/o estrategias que adopten definirán el éxito o fracaso universitario (Furlan, Heredia, Piemontesi y Sánchez, 2010).

Durante el proceso de la vida universitaria, el alumno presenta cambios individuales (crecimiento personal, académico y laboral) y cambios sociales (nueva red de soporte social). Por un lado, se evidencia la esperanza, el proyecto de vida, las motivaciones, etc., mientras que, por otro, evidenciamos las ilusiones, los temores, las angustias, etc. Además, se debe tener en cuenta que dicha experiencia significa un aprendizaje en cuanto a la autonomía y autorrealización del estudiante; quien, durante esta etapa, se hace cada vez más responsable de sus acciones; puesto que, comienza a valorar las consecuencias de la toma de decisiones (ámbito académico) que ha hecho anteriormente. Según Domínguez, Villegas y Centeno (2014), ingresar a la universidad plantea exigencias ante las cuales se requiere un afrontamiento exitoso para alcanzar los objetivos propuestos. Estas exigencias corresponden a situaciones que requieren a los universitarios organización del tiempo, elaboración de tareas, estudio frente a evaluaciones próximas, etc. En verdad, como señala Barraza (2007) muchos estudiantes pueden considerar estresante su paso por una institución académica.

Los autores difieren en cuanto a las estrategias de afrontamiento preferidas por los estudiantes. Figueroa y Estévez (2005) señalaron que los alumnos utilizan estrategias de afrontamiento dirigidos a solucionar el problema como tratar de concentrase, esforzarse, preocupación por el éxito, etc. Sin embargo, para Massone y Gonzáles (2003) los alumnos prefieren afrontar los quehaceres ligados a la educación con diversiones relajantes como leer, pintar, deporte, búsqueda de relaciones interpersonales en vez de buscar solución y fijarse en una meta.

Los estudiantes universitarios se ajustan a un nuevo contexto, que implica pasar de una etapa centrada en intereses personales (vida social) a una etapa centrada en conseguir objetivos para su proyecto de vida (Garzón y Gil, 2016). No obstante, frente a una tarea académica, los estudiantes universitarios pueden llegar a posponer la realización de la misma por diferentes razones, tales como dedicarse a actividades laborales o lúdicas. Algunos priorizan actividades caracterizadas como gratas, placenteras, divertidas por encima de otras que se califican como aburridas y desgastantes. Es por ello, que muchas veces atrasan o posponen actividades académicas frente a las no académicas. Este hábito, que se vuelve muy común en la vida universitaria, recibe la denominación de procrastinación. Según la Universidad del País Vasco (s.f.), el término procrastinar etimológicamente significa diferir, aplazar, posponer. La procrastinación es el hábito de postergar actividades consideradas como principales, siendo remplazadas por otras que son de carácter menos relevante o más agradable. En suma, la procrastinación consiste en realizar tareas diferentes a esa tarea principal que el sujeto sabe que debería estar haciendo, lo que genera consecuencias negativas. Como sostiene Guzmán (2014), la procrastinación constituye básicamente un problema de autorregulación y organización del tiempo; es decir, la persona que procrastina mantiene conductas de evasión.

Según Rigo (2014), existen diferentes factores por los cuales los adultos jóvenes caen en el hábito de procrastinar, como son el perfeccionismo, la naturaleza y la dificultad de la tarea y la impulsividad. Por ende, una persona es más proclive a procrastinar cuando la tarea es compleja, como es el caso de la carga universitaria.

Milgram (1988) planteó la importancia de diferenciar entre la procrastinación y la postergación intencional. Mientras que, en la procrastinación existe una postergación negligente; es decir, en este caso el individuo atrasa la ejecución de tareas por preferir otras actividades banales. En la postergación intencional, existe una planificación de labores; es decir los sujetos atrasan de manera estratégica. La diferencia entre una persona procrastinadora y una persona no procrastinadora radica en que la segunda planifica atrasar la tarea. Si la persona ha planificado atrasar la tarea por diferentes motivos, pero contempla realizarlo, es una persona no procrastinadora. Si la persona no ha gestionado su tiempo para realizar la tarea, es un caso de procrastinación (Natividad, 2014). En el contexto universitario es particularmente relevante la procrastinación académica. Según Senecal, Julien y Guay (2003), la procrastinación académica es una tendencia característicamente irracional que lleva a postergar ya sea el inicio o la culminación de una tarea académica. La procrastinación tiene su 
origen en el miedo al fracaso, la pobre gestión del tiempo, la aversión a la tarea, entre otros motivos (carácter multifacético). Como es de esperar, a mayor procrastinación, menor rendimiento académico (Natividad, 2014).

Rothblum, Solomon y Murakami (1986) consideran que la procrastinación académica está significativamente relacionada con el estrés personal y la salud física en estudiantes universitarios. Esta relación radica seguramente en los niveles de estrés provocados ante la necesidad de completar tareas cuya realización fue postergada y para lo cual el estudiante ya no dispone del tiempo necesario. Tales niveles de estrés, además, pueden tener efectos negativos en la salud en general del estudiante. Por otro lado, Gonzáles (2013) señala que la conducta procrastinadora se presenta cuando se van a realizar nuevas tareas y que, en algunos casos, es producto del miedo a la tarea que se debe llevar a cabo. A su vez, este miedo puede al menos en parte, verse facilitado por ciertas tendencias o predisposiciones del individuo como puede ser el caso de la ansiedad rasgo.

Spielberger (1972) señaló que es necesario establecer a la ansiedad como estado (estado afectivo de la persona) o como rasgo (dimensión de la personalidad). A partir de su teoría de la ansiedad estado-rasgo, postuló que cuando se habla de la ansiedad como estado emocional, nos referimos a una respuesta inmediata, modificable; donde hay una combinación de sentimientos como tensión, aprensión y nerviosismo. Por otro lado, cuando se estudia a la ansiedad como un rasgo de personalidad, nos referimos a las diferencias individuales de respuestas que pueden ser consideradas como estables; entendiendo a esta como una disposición, tendencia o rasgo. O, como describen Czernik, Giménez, Almirón y Larroza (2006): la ansiedad como rasgo es la predisposición de percibir circunstancias ambientales como amenazantes, mientas que la ansiedad como estado se refiere a la respuesta de la persona frente a una situación en específica, que se caracteriza por ser fluctuante y transitoria.

Entonces, cuando se evidencia un alto grado de ansiedad rasgo es porque los individuos consideran la mayoría de eventos como amenazantes; mientras que los niveles altos de ansiedad estado se interpretan como situaciones displacenteras episódicas, siendo lo más probable que estas personas generen estrategias de afrontamiento. Como sostienen De Ansorena, Cobo y Romero (1983), las variables estimulares influyen en la ansiedad estado, mientras que las variables personales influyen en la ansiedad rasgo.

Una serie de estudios indican de manera directa o indirecta que la procrastinación puede estar relacionada con la ansiedad. Rothblum et al. (1986) hallaron que los estudiantes procrastinadores tenían niveles altos de ansiedad frente a exámenes, seguidos de una autopercepción negativa acerca de sus estados afectivos. Ahora bien, se sabe que la procrastinación académica es muy recurrente en los estudiantes universitarios y gracias a estas investigaciones se puede sostener que este fenómeno no parece disminuir con el tiempo; vale decir que, conforme los universitarios escalan los grados de estudio, el nivel de procrastinación va aumentando y contribuyendo a un problema grave para los universitarios.

Asimismo, según Quant y Sánchez (2012), la procrastinación está relacionada con una baja autoestima, déficit de autoconfianza, déficit de autocontrol y comportamientos de desorganización, así como con las creencias que tiene la persona sobre sí misma. Ahora bien, debido a que estamos hablando dentro de un ámbito académico universitario; nos interesan todas aquellas creencias que el alumno se forma acerca de su papel como tal. Es ahí donde se observa que las autoevaluaciones de los estudiantes (metacognición) pueden influir en el hábito de procrastinar.

Flett, Blankstein, Hewitt y Koledin (1992) utilizaron un cuestionario para medir las cogniciones de los procrastinadores y pusieron en evidencia que la procrastinación está relacionada con la ansiedadrasgo, neuroticismo alto y responsabilidad baja. Asimismo, Flett, Stainton y Hewitt (2012) analizaron los pensamientos automáticos de los estudiantes procrastinadores aplicando el PCI (Procrastinatory Cognitions Inventory) y hallaron que la procrastinación está relacionada con cogniciones negativas en general y con algunas vinculadas con una actitud perfeccionista, todo lo cual indica niveles altos de ansiedad, entre otros aspectos.

Considerando dichos antecedentes, es pertinente plantearse si en el contexto peruano podemos verificar también una relación entre procrastinación académica y ansiedad rasgo, y específicamente en estudiantes universitarios. En el Perú, se han efectuado algunos estudios acerca de esta relación (e. g., Barreto, 2014; Vallejo, 2015), aunque estos trabajos investigaron con muestras de menores dimensiones que la del presente 
estudio. Algunos otros trabajos han considerado muestras de mayor tamaño, como es el caso del estudio de Durand y Cucho (2016), quienes investigaron en 306 universitarios peruanos, hallando una relación muy débil $(r=0.22)$ entre procrastinación académica y ansiedad, aunque el rango de carreras que abarcaron (Salud, Empresariales y Teología) fue más restringido que el de la presente investigación. Para el presente estudio se fijó como objetivo general determinar la relación entre la procrastinación académica y la ansiedad rasgo. Los objetivos específicos fueron investigar la posible relación entre las dimensiones de procrastinación académica (autorregulación académica y postergación de actividades) con la dimensión de ansiedad rasgo, así como establecer si existen diferencias en la procrastinación académica en función de las variables de edad, sexo, composición familiar, procedencia, facultad y cursos desaprobados.

\section{MATERIAL Y METODOS}

\section{Participantes}

La investigación tuvo una población objetivo conformada por 369 estudiantes universitarios pertenecientes al primer año de estudios de una universidad privada de Lima Metropolitana. De dicha población se tomó una muestra de 200 estudiantes. La Tabla 1 muestra las características sociodemográficas de dicho grupo.

Tabla 1. Distribución de la muestra del grupo de estudio según variables sociodemográficas

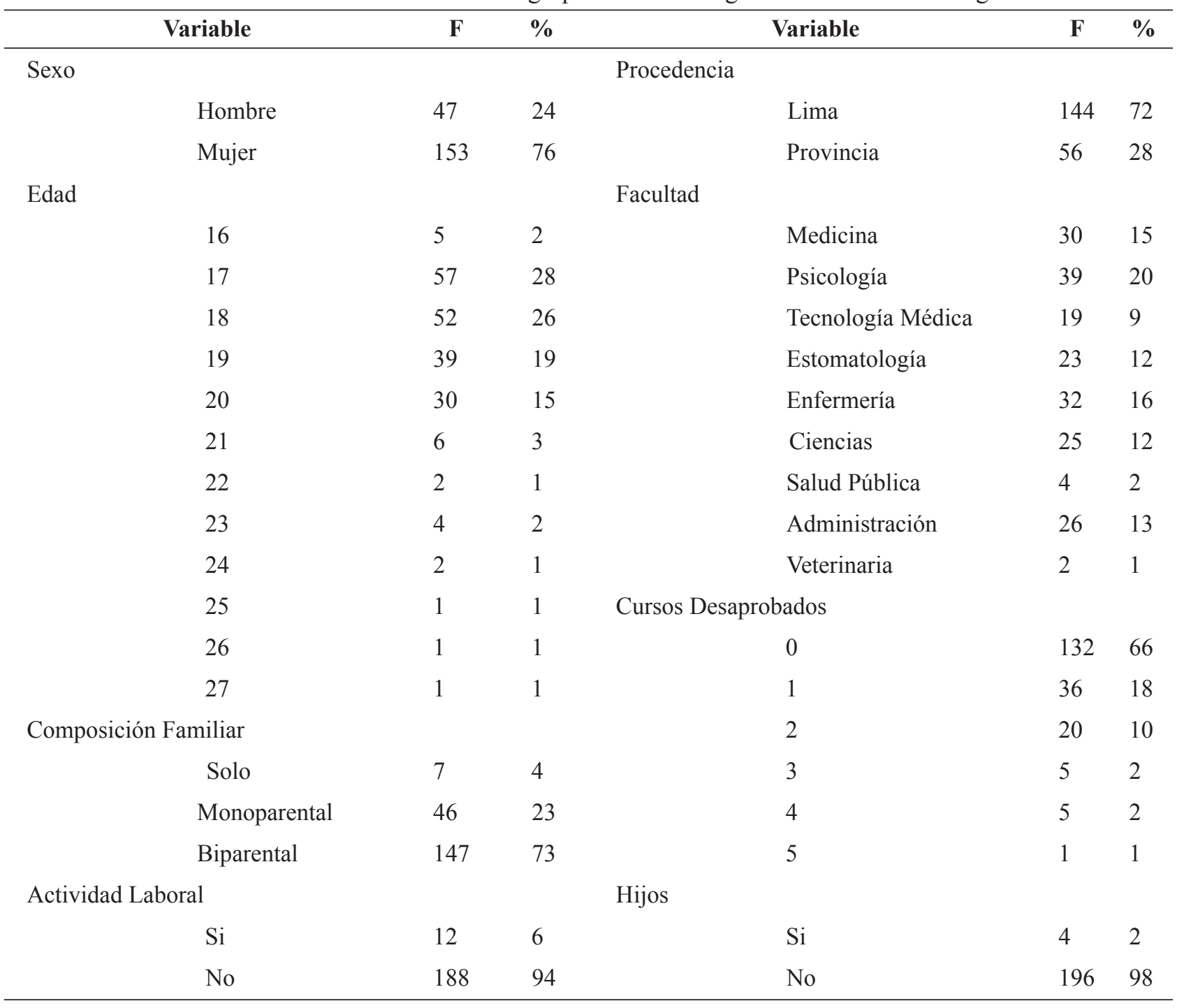

$\mathrm{n}=200$ 


\section{Instrumentos.}

Escalade Procrastinación Académica, EPA (Busko, 1998): Este instrumento cuenta con dos dimensiones: Autorregulación Académica y Postergación de Actividades. La primera dimensión cuenta con 9 ítems los cuales se califican de manera indirecta y la segunda dimensión cuenta con 3 ítems los cuales se califican de manera directa. Los ítems se responden en una escala Likert de cinco puntos. Las opciones de respuesta son: Nunca (1), Pocas Veces (2), A veces (3), Casi Siempre (4) y Siempre (5). La interpretación de los puntajes es directa; esto quiere decir que, a mayor puntaje obtenido de la prueba, el universitario presenta una conducta más elevada de procrastinación.

La EPA fue adaptada a la población universitaria de Lima por Domínguez et al. (2014) con una muestra constituida por 717 estudiantes universitarios de psicología que cursaban entre primer y décimo ciclo de estudios, de los cuales $74.3 \%$ fueron de sexo femenino, $33.5 \%$ estudiantes con actividad laboral y $92.2 \%$ estudiantes de estado civil soltero y cuyas edades oscilaban entre los 17 y 54 años. El análisis factorial confirmatorio indicó la presencia de los dos factores mencionados. Para determinar la confiabilidad se utilizaron los coeficientes: $\omega$ y $H$ debido al incumplimiento del supuesto de tauequivalencia de los ítems. En el caso de la dimensión de autorregulación académica se obtuvo $\omega=.892$ y $\mathrm{H}=.914$; mientras que en el caso de la dimensión de postergación de actividades se obtuvo $\omega=.811 \mathrm{y}$ $\mathrm{H}=.894$, siendo éstos coeficientes de confiabilidad elevados. Para la dimensión de autorregulación académica, puntuaciones menores a 27 indican un nivel bajo y puntuaciones mayores que 35 indican un nivel alto de procrastinación académica. Mientras que para la dimensión de postergación de actividades; puntuaciones menores a 7 indican un nivel bajo y puntuaciones mayores que 9 indican un nivel alto de procrastinación académica.

Inventario de Ansiedad Estado-Rasgo, IDARE (Spielberger y Díaz Guerrero, 1975). Es una escala breve para medir rasgos y estados de ansiedad. Cuenta con dos escalas independientes de autoevaluación de la ansiedad: estado (SXE) y rasgo (SXR). La escala de ansiedad rasgo (SXR); está compuesta por 20 ítems, de los cuales son 7 directos y 13 inversos. Y la escala de ansiedad estado (SXE) se compone de 20 ítems, de los cuales son 10 directos y 10 inversos. Cada uno de ítems es respondido a través de una escala Likert de cuatro puntos. Para la escala SXE las opciones de respuesta son: No en lo absoluto (1), Un Poco
(2), Bastante (3), Mucho (4). Para la escala SXR las opciones de respuesta son: Casi Nunca (1), Algunas Veces (2), Frecuentemente (3), Casi Siempre (4).

Este instrumento fue adaptado para la ciudad de Trujillo (Perú) por Castro (2015) en una muestra compuesta por 200 pacientes atendidos en una clínica privada. La muestra fue de pacientes mujeres con sobrepeso, con edades entre los 20 y 60 años. En el estudio de validez de contenido por criterio de jueces, ambas escalas obtuvieron coeficientes de Aiken entre 0,89 y 1,00 . Por otro lado, los 40 ítems correlacionaron de manera directa y significativa con sus respectivas escalas (coeficientes entre 0,255 y 0,640 en ansiedad rasgo y entre 0,322 y 0,687 en ansiedad estado. El análisis factorial confirmatorio con rotación varimax arrojó una estructura de dos factores. Para la validez concurrente, se utilizó la escala de ansiedad de Zung, obteniéndose correlaciones de 0,475 para la dimensión ansiedad estado y de 0,433 para ansiedad rasgo. Finalmente, en cuanto a validez clínica, el instrumento demuestra un $94 \%$ de sensibilidad, donde la prueba evidenció sus facultades de diagnosticar a un grupo "ansioso" y un $100 \%$ de especificidad, donde la prueba manifiesta sus facultades de determinar a un grupo "sin ansiedad" (Castro, 2015). Por otro lado, la confiabilidad de consistencia interna mediante alfa de Cronbach es bastante buena $(0,909$ en ansiedad estado y 0,873 para ansiedad rasgo). En cuanto a la consistencia por estabilidad, las correlaciones testretest fueron de 0,818 para ansiedad estado y 0,801 para ansiedad rasgo (Castro, 2015).

\section{Procedimiento.}

Para proceder a la recolección de datos se obtuvo el permiso del director general de la Unidad de Formación Básica de la universidad. Se entregó a cada alumno: el consentimiento informado, una ficha de datos sociodemográficos, la Escala de Procrastinación Académica (EPA) y el Inventario de Ansiedad Rasgo Estado (IDARE). Se procedió a realizar una base de datos codificados para mantener la confidencialidad de los participantes. Se elaboró una hoja de cálculo en un programa de dominio público y se transcribió los datos obtenidos incluyendo las variables sociodemográficas y las variables de comparación.

\section{Análisis de datos.}

Se utilizó la prueba de normalidad (prueba de normalidad de Kolgomorov-Smirnov), cuyos resultados evidenciaron que tanto los puntajes de 
la prueba de Escala de Procrastinación Académica como los del Inventario de Ansiedad Estado-Rasgo no presentan normalidad estadística. Por lo tanto, se procedió a usar pruebas no paramétricas. Se calculó la correlación canónica entre las puntuaciones totales de ambos instrumentos debido a la característica de las variables (multivariantes y dependientes). Se utilizó un programa de dominio público que facilitó el cálculo del coeficiente de correlación no lineal entre cada una de las dimensiones de cada instrumento administrado (EPA e IDARE). Se realizaron comparaciones en el nivel de procrastinación según edad, sexo, composición familiar, lugar de procedencia, facultad o carrera y número de cursos desaprobados. Para realizar estas comparaciones en variables dicotómicas se empleó la prueba $U$ de Mann-Whitney y en variables politómicas la prueba de Kruskal-Wallis.

\section{RESULTADOS}

La correlación canónica entre las puntuaciones totales de procrastinación y ansiedad fue de 0,068 , con que se concluye que la hipótesis planteada -la cual establece que hay una relación fuerte entre la procrastinación académica y ansiedad- no se acepta. Según Hernández, Fernández y Baptista (2010), el valor encontrado indica que la relación entre ambas variables es de tipo muy débil. Por otro lado, para la dimensión de autorregulación académica, el coeficiente de correlación no lineal fue de 0,152 ( $p=$ $0,033)$. Esto quiere decir que existe una relación de tipo muy débil, aunque estadísticamente significativa. Para la dimensión de postergación de actividades, el coeficiente de correlación no lineal fue de 0,158 ( $p=$ 0,172 ); dicho resultado permite concluir que no hay relación entre estas variables.

En la tabla 2 se aprecia que no existen diferencias significativas en la dimensión de autorregulación académica en función de las variables de "edad", "composición familiar", "facultad" y "cursos desaprobados". Del mismo modo, los resultados de la

Tabla 2. Prueba de Kruskal-Wallis de diferencias en autorregulación académica en función de la edad, composición familiar, facultad y cursos desaprobados.

\begin{tabular}{ccc}
\hline Variable & $\boldsymbol{K}$ & $\boldsymbol{p}$ \\
\hline Edad & 6,926 & .805 \\
Composición Familiar & 0,248 & .883 \\
Facultad & 6,493 & .592 \\
Cursos Desaprobados & 8,007 & .156 \\
\hline
\end{tabular}

Tabla 3 indican que no existen diferencias significativas en función de las mismas variables en la dimensión de postergación de actividades. En suma, las variables sociodemográficas consideradas (edad, composición familiar, facultad y número de cursos desaprobados) no parecen guardar relación con las dimensiones de procrastinación académica.

Tabla 3. Prueba de Kruskal-Wallis de diferencias en postergación de actividades en función de la edad, composición familiar, facultad y cursos desaprobados.

\begin{tabular}{ccc}
\hline Variable & $\boldsymbol{K}$ & $\boldsymbol{p}$ \\
\hline Edad & 16,153 & .136 \\
Composición Familiar & 0,579 & .749 \\
Facultad & 4,327 & .826 \\
Cursos Desaprobados & 7,984 & .157 \\
\hline
\end{tabular}

En cuanto a las diferencias en procrastinación académica según el sexo y el lugar de procedencia del participante, se estableció lo siguiente. La tabla 4 indica que existe una diferencia significativa en la autorregulación de actividades en función del sexo, pero no así en función del lugar de procedencia. Las mujeres presentan un mayor nivel de dificultades en la autorregulación. Asimismo, según los resultados de la Tabla 5, no existen diferencias en postergación de actividades en función ni del sexo ni del lugar de procedencia.

Tabla 4. Prueba U de Mann-Whitney de diferencias en autorregulación de actividades en función del sexo y lugar de procedencia

\begin{tabular}{ccc}
\hline Variable & $\boldsymbol{U}$ & $\boldsymbol{p}$ \\
\hline Sexo & 2889 & .041 \\
Procedencia & 3634 & .277 \\
\hline
\end{tabular}

Tabla 5. Prueba U de Mann-Whitney de diferencias en postergación de actividades en función del sexo y lugar de procedencia

\begin{tabular}{ccc}
\hline Variable & $\boldsymbol{U}$ & $\boldsymbol{p}$ \\
\hline Sexo & 3222 & 0,274 \\
Procedencia & 3870 & 0,654 \\
\hline
\end{tabular}

\section{DISCUSIÓN}

El principal resultado obtenido esa una correlación muy baja entre procrastinación académica y ansiedadrasgo. A pesar de lo previsto teóricamente, algunos estudios efectuados en estudiantes han arrojado 
correlaciones débiles (e. g., Barreto, 2014; Durand \& Cucho, 2016). Por ejemplo, Barreto (2014) encontró una relación positiva pero de tipo muy débil entre ambas variables en una investigación con 101 estudiantes en una universidad estatal en Lima. PardoBolívar, Perilla-Ballesteros y Salinas-Ramírez (2014), investigando en estudiantes colombianos de psicología, hallaron una correlación muy débil $(\mathrm{r}=0.23)$ entre la procrastinación académica y la ansiedad rasgo.

Resultados similares fueron obtenidos con otros instrumentos. Es el caso del estudio de Vallejos (2015) quien evaluó la ansiedad mediante la Escala de Reacción ante las Evaluaciones (/RTT) y sostuvo que mientras mayor sea la reacción ante las evaluaciones (ansiedad), mayor será la puntuación en la escala de procrastinación, a partir de una correlación moderada entre ambas variables. En cambio, estudios realizados en otras latitudes muestran que la ansiedad rasgo puede ser un predictor de la procrastinación, al menos dentro de un conjunto determinado de variables (Kamram \& Fatima, 2013).

Considerado los reportes en la literatura, se puede observar una amplia variabilidad en la magnitud de la correlación entre ansiedad rasgo y procrastinación en general o procrastinación académica. De acuerdo al planteamiento realizado en la introducción, podría sostenerse que las personas que presentan un mayor nivel de ansiedad presentarían altos niveles de procrastinación, indicando así una fuerte relación entre estas variables. Sin embargo, autores como McCow, Petzel y Rupert (citados en Guzmán, 2014), consideran que reducir el hecho de que las personas procrastinen sólo a un factor de ansiedad constituiría una visión muy limitada del fenómeno, ya que se sospecha que el hábito de procrastinar involucra también otros aspectos de personalidad. Es decir, la ansiedad puede mostrar una fuerte relación con la procrastinación en algunas poblaciones o bajo ciertas circunstancias, sin que esto pueda generalizarse a toda posible población. Otros factores que en algunos casos podrían tener relaciones más intensas con la procrastinación que la ansiedad son el estrés, la depresión, los estilos de afrontamiento, el neuroticismo, etc., (Guzmán, 2014). Se obtuvo correlaciones positivas, aunque débiles, entre las dimensiones de procrastinación y la ansiedad rasgo, pero de éstas sólo fue significativa la correlación entre autorregulación académica y ansiedad. Considerando que una mayor puntuación en autorregulación indica en realidad menos autorregulación, el resultado muestra una relación inversa entre la capacidad para autorregularse y la ansiedad rasgo. Este resultado se explica probablemente debido a las creencias irracionales del alumno, si consideramos que la procrastinación académica se caracteriza por ser un atraso irracional e intencional. Sin embargo, ambas correlaciones son bastante bajas, ratificando el hecho de que no se puede afirmar que en la población investigada se pueda verificar alguna relación entre procrastinación académica y ansiedad rasgo.

Se debe señalar el hecho de que los datos fueron obtenidos después de la entrega de exámenes de los alumnos. Furlán, Sánchez, Heredia, Piemontesi e Illbele (2009) señalan que la ansiedad es evidente en los estudiantes universitarios frente a un estímulo específico estresor como es el caso de los exámenes; una vez que estos desaparecen de la percepción del alumno, los universitarios dejan de tener conductas de carácter ansioso. Los universitarios presentan niveles altos de ansiedad estado, gracias al estímulo de los exámenes. Al momento que desaparece el estímulo de los exámenes, la conducta ansiosa se disipa tanto en alumnos procrastinadores como no procrastinadores. No obstante, durante las fechas de exámenes altos niveles de ansiedad estado podrían presentarse en estudiantes con niveles de ansiedad rasgo bajos. Ambos factores de ansiedad tienen dinámicas distintas y los resultados apuntan a que niveles bajos o altos de ansiedad rasgo pueden estar presentes en estudiantes procrastinadores y no procrastinadores.

Los resultados también mostraron que no existen diferencias en las dimensiones de la procrastinación académica en función de variables como la edad, composición familiar, carrera, número de cursos desaprobados y lugar de procedencia y que sólo en la dimensión de autorregulación se halló una diferencia según sexo. Es sabido que las variables sociodemográficas impactan de diversa manera en los factores psicológicos y la procrastinación no es una excepción a esta regla. Como indicaron PardoBolívar, Perilla-Ballesteros y Salinas-Ramírez (2014), para la procrastinación académica no existe un perfil propiamente dicho.

Algunos otros estudios también indican mayor procrastinación en las mujeres. Carranza y Ramírez (2013) investigaron en 302 estudiantes de una universidad privada en Tarapoto (Perú) y hallaron un perfil de procrastinador con las características siguientes: sexo femenino entre los 16 y 20 años de edad y perteneciente a la facultad de ingeniería, arquitectura o de salud. 
Sin embargo, en otros estudios, se encuentra más procrastinación entre los varones y éste parece ser el resultado más común. En el Perú, Chávez (2015) investigó en 118 universitarios entre los 18 y 28 años de edad de las facultades de ciencias e ingeniería y gestión y alta dirección y halló procrastinación principalmente en varones. Otro estudio, igualmente en universitarios en el Perú encontró también más procrastinación en estudiantes de sexo masculino (Chan, 2011). En otros países se ha encontrado resultados similares. Balkis y Duru (2009) también hallaron, en universitarios de Turquía, que los hombres procrastinan más que las mujeres; además que la procrastinación mantiene una relación inversa con la variable edad. Por supuesto, las diferencias entre los resultados reportados en la literatura y las de éstos con los del presente estudio pueden deberse a factores contextuales bastante específicos de cada ambiente, momento y circunstancias, tanto de la población investigada, como del acto mismo de evaluación, todo esto incluyendo factores culturales como de diferencias en los instrumentos empleados.

\section{REFERENCIAS}

Balkis, M. \& Duru, E. (2009). Prevalence of academic procrastination behavior among pre-service teachers, and its relationship with demographics and individual preferences. Journal of Theory and Practice in Education, 5(1): 18-32.

Barraza, A. (2007). Estrés académico: un estado de la cuestión. Revista Psicología Científica.com. Recuperado de:http://www.psicologiacientifica.com/estresacademico-2

Barreto, M. (2014). Relajación en estados de ansiedad y procrastinación en ingresantes a la Facultad de Ciencias Sociales 2015. Universidad Nacional de Tumbes.(Tesis para optar el Grado académico de Doctora en Psicología. Lima, Perú: Universidad Nacional Mayor de San Marcos).

Busko, D. (1998). Causes and consequences of perfectionism and procrastination: a estructural equation model (Tesis de maestría no publicada. Guelph, Ontario: University of Guelph).

Carranza, R. \& Ramírez, A. (2013). Procrastinación y características demográficas asociados en estudiantes universitarios. Apuntes Universitarios, 3(2), 95-108.

Castro, I. (2015). Propiedades psicométricas del inventario de ansiedad: Rasgo-estado (IDARE) en pacientes que asisten a la clínica de control de peso Isell Figura Vital de Trujillo, 2015. (Tesis para optar el grado académico de Doctora en Psicología, Trujillo, Perú, Universidad César Vallejo).

Chan, L. (2011). Procrastinación académica como predictor en el rendimiento académico en jóvenes de educación superior. Temática Psicológica, 7(1), 53-62.

Chávez, E. (2015). Procrastinación crónica y ansiedad estado-rasgo en una muestra de estudiantes universitarios. (Tesis para optar el título de Licenciado en Psicología con mención en Psicología Clínica, Lima, Perú, Especialidad de Psicología, Facultad de Letras y Ciencias Humanas, Pontificia Universidad Católica del Perú).

Czernik, G., Giménez, N., Almiróns, L. \& Larroza, G. (2006). Ansiedad rasgo-estado en una Escuela de Formación Profesional de la ciudad de Resistencia (Chaco). Corrientes, Argentina: Departamento de Salud Mental, Medicina Legal y Salud Pública, Universidad Nacional del Nordeste.

De Ansorena, A., Cobo, J. \& Romero, I. (1983). El constructo ansiedad en Psicología: una revisión. Estudios en Psicología, 16, 31-45.

Domínguez, S., Villegas, G. \& Centeno, S. (2014). Procrastinación académica: validación de una escala en una muestra de estudiantes de una universidad privada. Liberabit, 20(2), 293-304.

Durand, C. E., \& Cucho, N. S. (2016). Procrastinación académica y ansiedad de una universidad privada de Lima Este, 2015. (Tesis para optar el Título Profesional de Psicóloga. Lima, Perú, Universidad Peruana Unión. Figueroa, M. \& Estévez, A. (2005). Las estrategias de afrontamiento y su relación con el nivel de bienestar psicológico. Un estudio con adolescentes de nivel socioeconómico bajo de Tucumán (Argentina). Anales de Psicología, 21(1), 66-72.

Flett, G.L., Blankstein, K.R., Hewitt, P.L. \& Koledin, S. (1992). Components of perfectionism and procrastination in college students. Social Behavior and Personality, 20(2), 85-94.

Flett, G. L., Stainton, M. \& Hewitt, P. (2012). Procrastination automatic thoughts as a personality construct: An analysis of the procrastinatory cognitions inventory. Journal of Rational Emotive and Cognitive Behavior Therapy, 30, 223-236.

Furlan, L. A., Heredia, D., Piemontesi, S. \& Sánchez, J. (2010). Procrastinación y ansiedad frente a los exámenes en estudiantes de psicología de la UNC. En: II Congreso Internacional de Investigación y Práctica Profesional en Psicología XVII Jornadas de Investigación Sexto Encuentro de Investigadores en Psicología del MERCOSUR. Buenos Aires, Argentina.

Furlan, L. A., Sánchez, J., Heredia, D., Piemontesi, S. \& Illbele, A. (2009). Estrategias de aprendizaje y ansiedad ante los exámenes en estudiantes universitarios. Pensamiento Psicológico, 5(12), 117-123.

Garzón, A. \& Gil, J. (2016). El papel de la procrastinación académica como factor de la deserción universitaria trabajo. Revista Complutense de Educación, 28(1), 307324.

González, L. (2013). La ansiedad lleva a postergar 
el trabajo. Lideres. Recuperado de: http://www. revistalideres.ec/lideres/ansiedad-lleva-postergar.html Guzmán, D. (2014). Procrastinación: una mirada clínica. Barcelona, España: Universidad de Barcelona.

Hernández, R., Fernández, C. \& Baptista, P. (2010). Metodología de la investigación. Ciudad de México: McGraw-Hill.

Kamram, W. \& Fatima, I. (2013). Emotional intelligence, anxiety and procrastination in intermediate science students. Pakistan Journal of Social and Clinical Psychology, 11(2), 3-6.

Massone, A. \& González, G. (2003). Estrategias de afrontamiento (coping) y su relación con el logro académico en matemática y lengua en adolescentes de noveno año de educación general básica. Revista Iberoamericana de Educación, 3(2), 00. Recuperado de: https://rieoei.org/RIE/article/view/2972/3887

Milgram, N. (1988). Procrastination in daily living. Psychological Reports, 63, 752-754.

Natividad, L. A. (2014). Análisis de la procrastinación en estudiantes universitarios. ( Tesis doctoral. Facultat de Psicologia, Universitat de València, Valencia, España.

Pardo-Bolívar, D., Perilla-Ballesteros, L. \& SalinasRamírez, C. (2014). Relación entre procrastinación académica y ansiedad-rasgo en estudiantes de psicología. Cuadernos Hispanoamericanos de Psicología, 14(1), 3144.

Quant, D. \& Sánchez, A. (2012). Procrastinación, procrastinación académica: concepto e implicaciones. Revista Vanguardia Psicológica Clínica, Teórica y Práctica, 3(1), 45-59.
Rigo, E. (2014). Cómo reducir la procrastinación, trabajando la ansiedad. MEDCIENCIA.

Recuperado de: https://es.sott.net/article/28133Como-reducir-la-procrastinacion-trabajando-laansiedadRothblum, E. D., Solomon, L. J. \& Murakami, J. (1986). Affective, cognitive, and behavioral differences between high and low procrastinators. Journal of Counseling Psychology, 33(4), 387-394.

Senecal, C., Julien, E. \& Guay, F. (2003). Role conflict and academic procrastination: a self-determination perspective. European Journal of Social Psychology, 33(1), 135-145.

Spielberger, C. D. (1972). Needed research on stress and anxiety. A special report of the USOE-Sponsored Grant Study: Critical Appraisal of Research in the PersonalityEmotions-Motivation Domain. IBR Report No. 72-10. Texas; USA: Texas Christian Univ. Recuperado De: https://files.eric.ed.gov/fulltext/ED113649.pdf

Spielberger, C.D. \& Díaz-Guerrero, R. (1975). Idare: Inventario de ansiedad rasgo-estado. Ciudad de México: El Manual Moderno.

Universidad del Pais Vasco. (s.f.).Yo procrastino, tú procrastinas....Lejona: Universidad del Pais Vasco. Recuperado de: https://www.ehu.eus/ documents/2632144/2634184/Yo+procrastino.pdf/ ab93b6fd-7f22-418d-9af6-e0d1bcb9ed0a

Vallejos, S. (2015). Procrastinación académica y ansiedad frente a las evaluaciones en estudiantes universitarios. (Tesis para optar el título de Licenciada en Psicología. Lima, Perú, Pontificia Universidad Católica del Perú). 